\title{
Prolonged use of a proton pump inhibitor reduces microbial diversity: implications for Clostridium difficile susceptibility
}

\author{
Charlie T Seto ${ }^{1,2}$, Patricio Jeraldo ${ }^{2,3}$, Robert Orenstein ${ }^{4}$, Nicholas Chia ${ }^{1,2,5,6,7^{*}}$ and John K DiBaise ${ }^{*^{*}}$
}

\begin{abstract}
Background: The role of the gut microbiome in arresting pathogen colonization and growth is important for protection against Clostridium difficile infection (CDI). Observational studies associate proton pump inhibitor (PPI) use and CDI incidence. We hypothesized that PPI use affected the distal gut microbiome over time, an effect that would be best explored by time-longitudinal study of healthy subjects on PPI in comparison to treatment-naive CDI subjects. This study enrolled nine healthy human subjects and five subjects with treatment-naive CDI. After random assignment to a low $(20 \mathrm{mg} /$ day $)$ or high $(2 \times 20 \mathrm{mg} /$ day) dose group, fecal samples were collected from the nine healthy subjects before, during, and after 28 days of PPI use. This was done in conjunction with pre-treatment fecal collection from CDI subjects. High-throughput sequencing ( $16 \mathrm{~S}$ rRNA) was performed on time-longitudinal samples to assess changes to the healthy gut microbiome associated with prolonged PPI usage. The healthy samples were then compared to the CDI subjects to explore changes over time to the gut microbiome associated with PPI use and potentially related to CDI.
\end{abstract}

Results: We report that PPI usage at low and high dosages, administered for 28 days, resulted in decreases to observed operational taxonomic unit (OTU) counts after both 1 week and 1 month. This decrease resulted in observed OTU levels that were similar to those found in treatment-naive CDI patients, which was partly reversible after a 1 month recovery period. We did not detect a dose-dependent difference in OTU levels nor did we detect significant changes in taxa previously reported to be affected by PPI treatment.

Conclusion: While our observation of diminishing observed OTU counts during PPI therapy is a preliminary finding in a small cohort, our hypothesis that PPIs disrupt the healthy human gut microbiome is supported in this group. We conclude that decreases in observed species counts were reversible after cessation of PPI usage within 1 month. This finding may be a potential explanation for the association between prolonged PPI usage and CDI incidence.

Keywords: Proton pump inhibitor, Gut microbiome, Clostridium difficile

\section{Background}

Proton pump inhibitors (PPIs) are potent inhibitors of gastric acid production that are highly effective for treating acid-mediated disorders of the upper digestive tract [1]. PPIs are one of the most commonly prescribed medications in the USA, with billions of dollars in annual sales [2]. Although they have a long history of safety and efficacy

\footnotetext{
* Correspondence: Chia.Nicholas@mayo.edu; DiBaise.John@mayo.edu ${ }^{1}$ Biomedical Informatics and Computational Biology, University of Minnesota-Rochester, Rochester, MN, USA

${ }^{2}$ Center for Individualized Medicine, Mayo Clinic, Rochester, MN, USA

${ }^{8}$ Division of Gastroenterology, Mayo Clinic, Scottsdale, AZ, USA Full list of author information is available at the end of the article
}

[3], epidemiological studies have linked PPIs to several nutritional, metabolic, and infectious disorders. Specifically, their prolonged use has been associated with iron [4] and vitamin B12 [5,6] deficiencies, hypomagnesemia [7], osteoporosis-related fractures [8,9], small intestinal bacterial overgrowth $[10,11]$, and community-acquired pneumonia [12,13]. Furthermore, several recent studies have found an association between the use of PPIs and the development of Clostridium difficile infection (CDI) [14-18]. A meta-analysis of 39 studies reported a 1.74-fold increase in risk of CDI in PPI users (95\% confidence interval 1.472-05) [19]. This association with the most common 
healthcare-acquired infection led the U.S. Food and Drug Administration (FDA) to require that the package insert for PPIs contain a warning that PPIs may increase the risk of CDI [20].

Despite this epidemiological association, the reason why PPIs might increase CDI risk is not known. Although hypochlorhydria increases susceptibility to enteric bacterial infections, $C$. difficile spores are unaffected by gastric acidity [21]. The intestinal microbiota play an important function in suppressing pathogen growth (i.e., colonization resistance) [22], and alterations in the microbiota by PPIs may induce $C$. difficile proliferation. The loss of colonic microbial diversity is a characteristic feature of CDI [23,24]. Microbiome analysis of the feces has been shown to improve the ability to distinguish CDI status when comparing cases with both diarrheal and non-diarrheal controls [25]. Prolonged use of PPIs has been linked to changes in the microbial community composition of the upper intestinal tract in vitro [10,11,26,27]. Gastric acid reduction may also influence the microbial composition of the lower gastrointestinal (GI) tract [26]. The longitudinal changes in the gut microbial ecology during and after PPI use and how these relate to the microbiota present in CDI remain unknown.

The purpose of this study was to investigate the impact of short- and long-term PPI use on the fecal microbiota and to compare these changes to persons with newly diagnosed CDI. If similar, it would provide a potential mechanism for the association of PPIs with an increased risk of CDI. Changes to the fecal microbial community induced by PPI use were assessed in healthy humans using highthroughput $16 \mathrm{~S}$ hypervariable tag sequencing and compared to patients with a treatment-naïve first episode of CDI. Our results demonstrate that microbial diversity declines following prolonged (28-day) PPI usage. This decrease results in observed operational taxonomic unit (OTU) levels that are similar to those found in treatmentnaïve CDI patients. The reduction in observed OTU levels was not always reversible 1 month after ceasing treatment.

\section{Results}

\section{Study participants}

Ten healthy volunteers (five women, five men) were randomly assigned to receive omeprazole $20 \mathrm{mg}$ once daily $(n=5)$ or twice daily $(n=5)$ for 28 days. Volunteers ranged in age from 18 to 57 years (mean \pm standard deviation: $40.8 \pm 15.3$ years), with body mass indices (BMI) ranging from 18.6 to $29.7 \mathrm{~kg} / \mathrm{m}^{2}$ (mean \pm standard deviation: $24.8 \pm 3.7 \mathrm{~kg} / \mathrm{m}^{2}$ ). Stool samples were collected prior to baseline (T0) and following 7 days (T7) and 28 days (T28) of omeprazole use. A final stool sample was collected 1 month after discontinuation of omeprazole (T56). Fecal DNA was sequenced and compared to that from five treatment-naïve CDI patients, all women, ranging in age from 20 to 63 years (mean \pm standard deviation: $40.8 \pm 15.9$ years) with BMI ranging from 16.6 to $39.4 \mathrm{~kg} / \mathrm{m}^{2}$ (mean \pm standard deviation: $28.1 \pm 8.7 \mathrm{~kg} / \mathrm{m}^{2}$ ).

No adverse events were reported amongst the healthy subjects receiving omeprazole. One healthy subject was withdrawn from the study after providing the baseline and the first on-PPI stool samples due to subsequent antibiotic use prescribed by his primary care provider.

The OTUs corresponding to Clostridium in CDI subjects were assessed to determine if $C$. difficile could be detected across all five subjects. C. difficile BI1 was detected in 4/5 CDI subjects (as OTU 57). Other major Clostridium associated with CDI subjects included Clostridium butyricum (as OTU 377) in 4/5 CDI subjects and Clostridium perfringens (as OTU 393) in 3/5 CDI subjects.

\section{Sequencing}

The total number of utilized read-pairs per sample from healthy volunteers and CDI patients was 14,635,736 (mean \pm standard deviation $340,366 \pm 500,176$ ). The lowest read-count of a sample was 75,506 (see Additional file 1: Metadata). Rarefaction plots measuring observed-OTU diversity indicate that all samples were sequenced past exponential species accumulation (Additional file 2: Figure S1).

\section{PPI use is associated with reduced OTU diversity}

After rarefaction of all samples to 75,000 reads, a significant difference in observed OTU count between baseline and 1 month was observed (Figure 1, Table 1). To determine the robustness of this observed difference to sampling depth, sensitivity analysis was performed by generating additional rarefactions of 50,000 and 25,000 reads/sample to assess the effect of reduced read depth (Additional file 2: Figure S2). The significant change in observed species count between baseline and 1 month on PPI remained significant at 50,000 and 25,000 reads/sample (Additional file 2: Table S1).

\section{Microbial diversity differences between genders in PPI users}

After dividing subjects by gender and comparing observed OTU counts across all time points, a statistically significant difference between observed OTU counts in men $(n=4)$ and women $(n=5)$ on PPIs was found $(p \approx 0.004$, Additional file 2: Figure S3). However, when observed OTU counts at each time point (T0, T7, T28, T56) for men and women were compared, the differences observed were not significant $(p \approx 0.10, p \approx 0.21, p \approx 0.10, p \approx 0.10)$. For observed OTU counts, the mean \pm standard deviation for women was $226 \pm 55$ and $293 \pm 77$ for men.

\section{No PPI dose-dependent effect on diversity}

A comparison of OTU counts between the high (twice daily PPI) and low (once daily PPI) dose groups revealed no significant difference in observed OTU counts (i.e., 


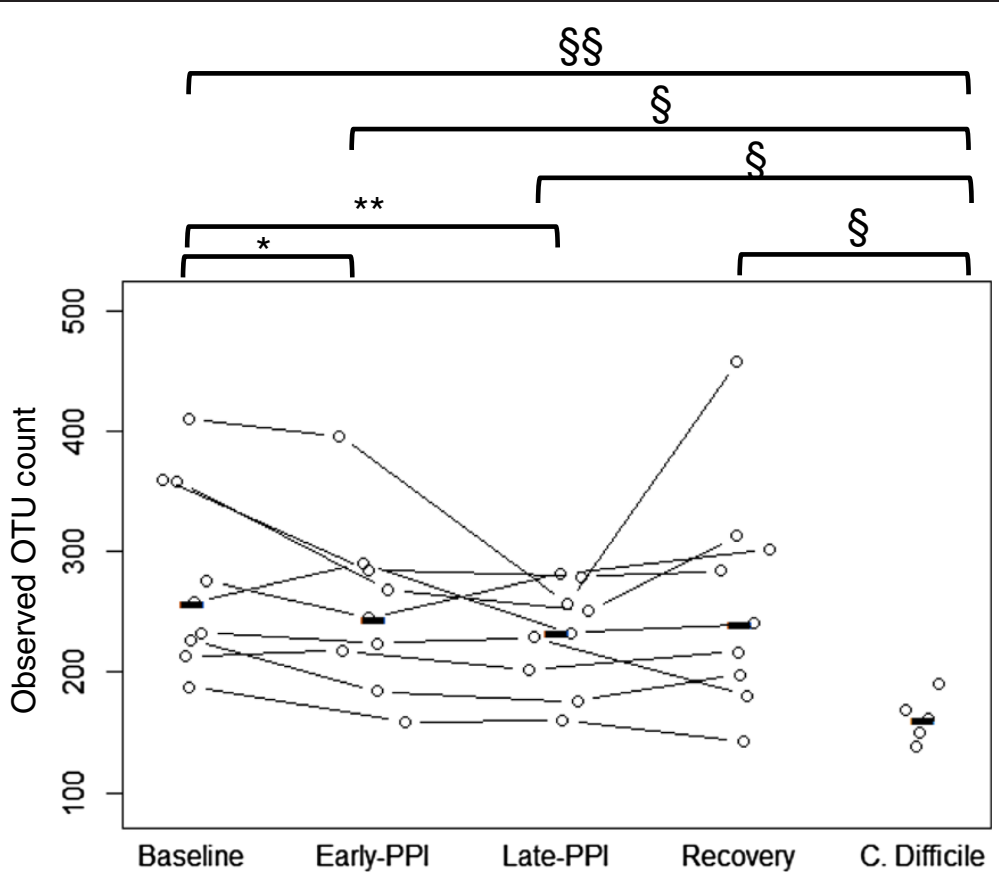

Figure 1 Observed OTU counts on proton pump inhibitors. Plot depicts patient observed-OTU counts at start of treatment, 1 week into treatment, and at the end of the 1-month program, followed by final collection 1 month after ceasing PPI usage. Five first-incidence C. difficile patients had stool isolated and sequenced to represent the $C$. difficile cohort. Black horizontal bars represent within-group means. All statistical tests using matched-pair healthy volunteers used Wilcoxon signed rank; statistical tests between healthy volunteer groups $\left({ }^{*} p<0.05,{ }^{* *} p<0.005\right)$ and $C$. difficile group used Wilcoxon rank sum $(\$ p<0.05, \S \S p<0.01)$.

prior to omeprazole use) between the groups at baseline, 7 days on PPI, 28 days on PPI, or 1 month after stopping the PPI $(p \approx 0.2778, p \approx 0.2063, p \approx 0.5556, p \approx 0.2778$, respectively). After separating subject time points into two groups based on dosage, no significant difference was detected between the 20-mg once/day and 20-mg twice/day groups $(p \approx 0.072)$; mean \pm standard deviation for the once daily omeprazole group was $239 \pm$

Table 1 Statistical significance of observed OTU changes between sample groups

\begin{tabular}{ccccc}
\hline Cohort & T0 & T7 & T28 & T56 \\
\hline T0 & & & & \\
T7 & 0.0117 & & & \\
T28 & 0.0039 & 0.0328 & & \\
T56 & 0.0328 & 0.9102 & 0.1069 & \\
CD & 0.0051 & 0.0112 & 0.0829 & 0.0599 \\
\hline
\end{tabular}

Table of statistical tests between matched-pair healthy subject time intervals to determine the significance of measured differences in observed species counts. Species counts were calculated from samples rarefied to 75,000 reads. Testing for matched-pair healthy samples used one-tailed (alternative = "greater") Wilcoxon signed rank

${ }^{a}$ Non-matched pair probabilities for groups with C. difficile (CD) were calculated using one-tailed (alternative $=$ "greater") Wilcoxon rank sum with continuity correction.
62 and for the twice daily omeprazole group was $277 \pm$ 81 (Additional file 2: Figure S4).

\section{Longitudinal gut microbe composition is largely stable}

After rarefaction of all samples to 75,000 reads, the percent of OTUs that remained throughout the course of the PPI therapy varied between $14.33 \%$ and $37.38 \%$ (median \pm MAD of $25.3 \% \pm 9.5 \%$ ) across the nine subjects, corresponding to longitudinal trend 1111 (Additional file 2: Table S2). Of the total OTUs observed in the subjects, $4.08 \%-14.13 \%$ (Median \pm MAD of $7.7 \% \pm 1.6 \%$ ) appeared after the conclusion of the PPI therapy, corresponding to longitudinal trend 0001.

\section{Similarity of OTU richness between PPI use and CDI}

Using subject data rarefied to 75,000 reads/sample, observed OTU diversity was compared between the five CDI subjects as well as the time points of male or female subjects (Additional file 2: Table S3). The change in observed OTUs in CDI subjects and all healthy subjects were also compared by time interval (Table 1). We reported marked differences in observed OTU counts between healthy subjects at baseline and those with CDI that erode after 1 week and 1 month of PPI therapy (Table 1). This difference in observed OTU counts is also apparent when the healthy 
subjects are subdivided into male and female groups (Additional file 2: Table S3).

\section{No compositional overlap between PPI use and CDI}

Non-metric multidimensional scaling (NMDS) and SourceTracker were used to determine if healthy subjects using a PPI retained microbial ecological similarity to baseline samples or if a CDI-like microbial composition could be detected. Compositional similarity to the CDI patients was potentially indicated in one of the subjects (D02d, Figure 2). The NMDS plots showed strong overlap of on-PPI time points (Figure 2). A SourceTracker calculation indicated the closest sink to on-PPI or afterPPI samples was baseline compared to the five CDI subjects, and an "Unknown" group as visualized in a ternary plot (Additional file 2: Figure S5).

\section{No detectable PPI-induced pathway changes}

The nearest sequenced taxon index (NSTI), a metric to determine divergence of taxonomic reads from closed references, was calculated per sample with mean \pm standard deviation of $0.07 \pm 0.03$. These reported values are within the range of the NSTI generated from the phylogenetic investigation of communities by reconstruction of unobserved states (PICRUSt) benchmarking of mammalian gut samples $(0.14 \pm 0.06)$ [28]. No significant change in enrichment in any of the Kyoto Encyclopedia of Genes and
Genomes (KEGG) pathways was detected during PPI treatment of healthy subjects. However, there was a significant enrichment in glycine-serine-threonine metabolism in healthy patients pre-PPI use compared to the CDI subjects $(p \approx 0.001$, Figure 3 , Additional file 2 : Table $S 4$ ). The enrichment of this pathway decreased during the course of PPI treatment until it was not significantly different from the CDI group, and the enrichment of this pathway increased during recovery until significantly it was different from the CDI subjects $(p \approx 0.007$, Figure 3, Additional file 2: Table S4).

\section{Individual ecological distinctiveness is preserved}

The unweighted UniFrac $\beta$-diversity distance matrix was calculated from a sample rarefied to 75,000 reads/sample (Figure 4), with one subject samples remaining most similar to each other for the duration of treatment (subjects 4). Three subjects had their 1-month sample as the outlier (subjects 6, 8). Four CDI subjects (C. difficile (CD) subjects $1,2,4)$ clustered closely to each other, with CDI subject 3 forming a sub-cluster with healthy subject 1's post-treatment sample (D01d) and CDI subject 5 clustering with subject 3 at baseline (D03a).

\section{Discussion}

We conducted a study that longitudinally tracked the fecal microbiome of healthy humans before, during, and

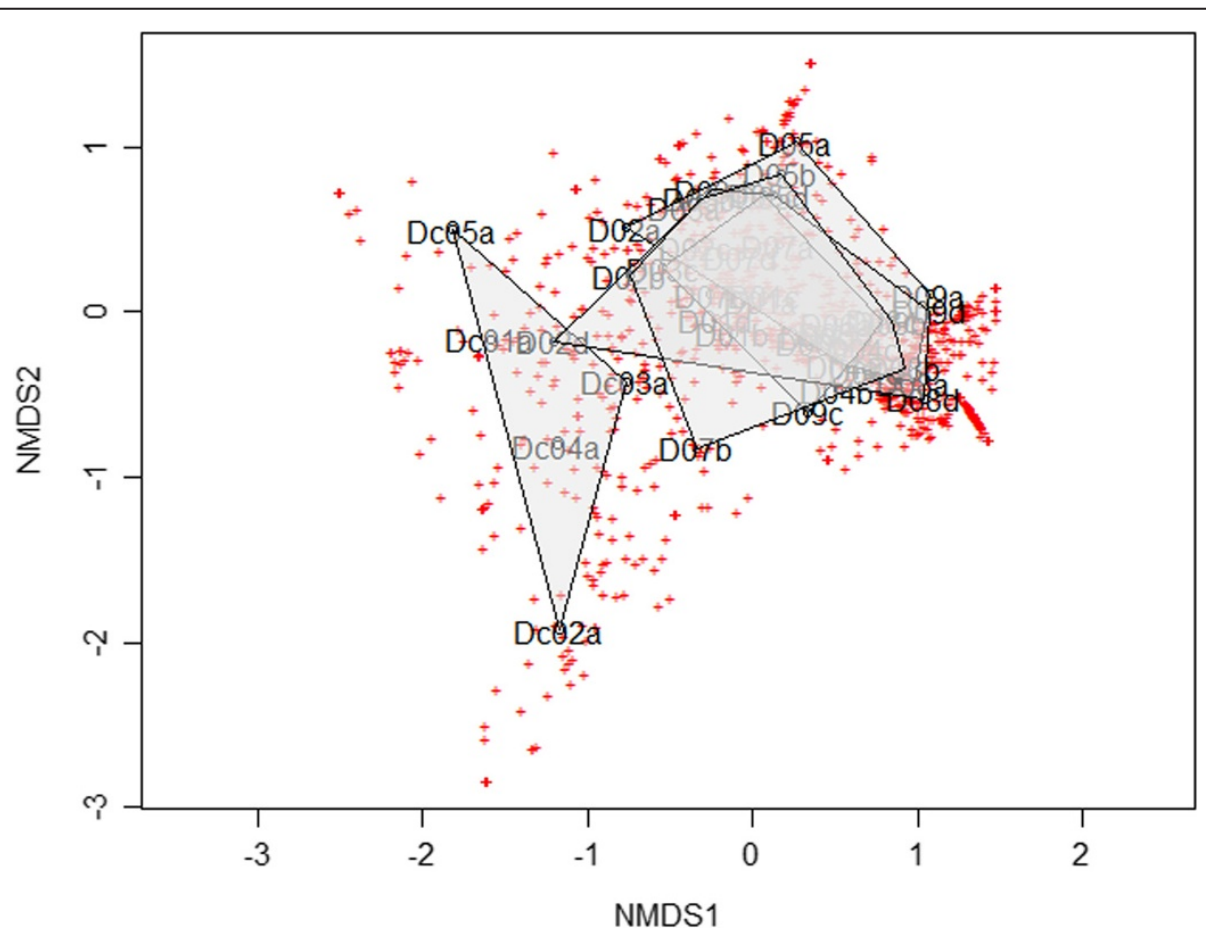

Figure 2 No overlap of on-PPI and CDI subjects. NMDS plots show no taxonomic overlap between short- and long-term PPI users and subjects with C. difficile infection, with strong overlap between all subjects and all time points. 


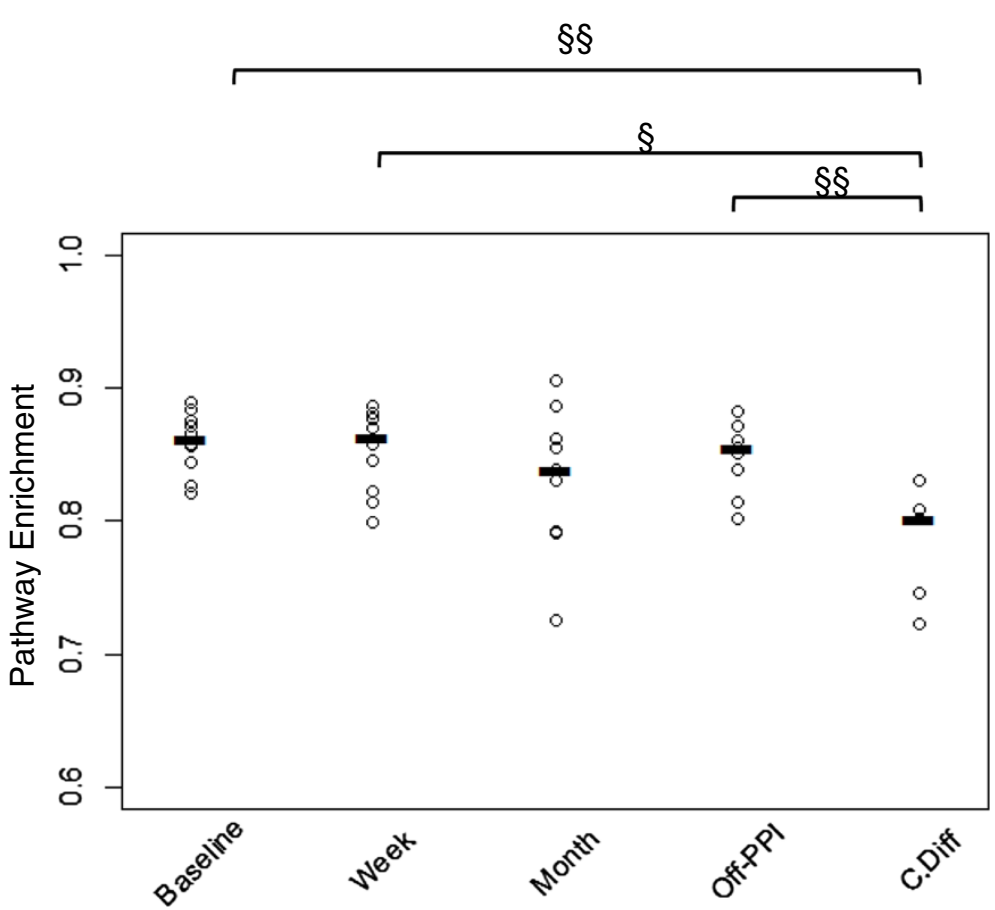

Figure 3 KEGG Pathway disruptions on PPI. Plot depicts relative enrichment of KEGG pathway "Glycine, Serine, Threonine Metabolism" at start of treatment, one week into treatment, and at the end of the one-month program. The difference in relative abundance of the pathway for the duration of treatment did not vary significantly, with significant difference in means between subjects with $C$. difficile and other time points except the one-month group $(\S p<0.05 ; \S \S p<0.005)$.

1 month after a once- or twice-daily PPI use to investigate the hypothesis that PPI use can cause perturbations to the healthy gut microbiome that bear similarity to the microbial changes that occur in CDI. While limited by a small sample size, we observed that PPIs induce a marked and persistent decrease in observed OTU count, a surrogate for microbial diversity. At the end of the 28-day treatment period, the observed OTU counts were similar to those from patients with a first CDI episode indicating the potential for PPIs to negatively influence the robustness of the host microbial ecology and increase the susceptibility to CDI.

This is the first study to examine the impact of PPI use on the human gut microbiota using high-throughput sequencing. Our findings are consistent with past longitudinal studies of the GI microbiome that indicate stable individual microbial communities [29]. That is to say, despite the effects of PPI use, samples from the same subject taken over time remained more similar to each other than to the samples obtained from the other healthy subjects. In our work, this is supported by the unweighted UniFrac $\beta$-diversity distance matrix (Figure 4) showing clusters comprised of individual samples. NMDS plots also show minimal movement of individual samples over time, despite the overall reduction in diversity over time with PPI use.
Garcia-Mazcorro et al. showed that PPI usage in canines resulted in an increase of Lactobacillus spp. in male $(n=4)$ and female dogs $(n=4)$ and a decrease in Faecalibacterium spp. and Bacteroides-Prevotella-Porphyromonas in the male dogs only [30]. In contrast, our comparison of taxa highlighted in Garcia-Mazcorro et al. revealed no significant differences during PPI treatment (Additional file 2: Table S5). The lack of taxa-specific associations in our human cohort may be due to the larger baseline differences that arise due to differences in age, environment, diet, and starting microbiomes, effects that are more difficult to overcome with small sample sizes. This variability can be observed in changes of relative abundance of taxa over time that varies between subjects (Additional file 2: Figure S6). Garcia-Mazcorro et al. also collected mucosal biopsy samples from the stomach and duodenum of the dogs, reporting a decrease in Helicobacter spp. and a corresponding increase in the relative abundance of other bacteria in the stomach. However, in the duodenum, an overall increase in the abundance of all bacteria (i.e., small bowel bacterial overgrowth) was seen. Garcia-Mazcorro et al. did not report major qualitative changes in the phylogenetic composition of stomach and duodenal microbial communities after PPI use. A limitation of our study is that, given the complexities and expense of obtaining specimens from the upper GI 


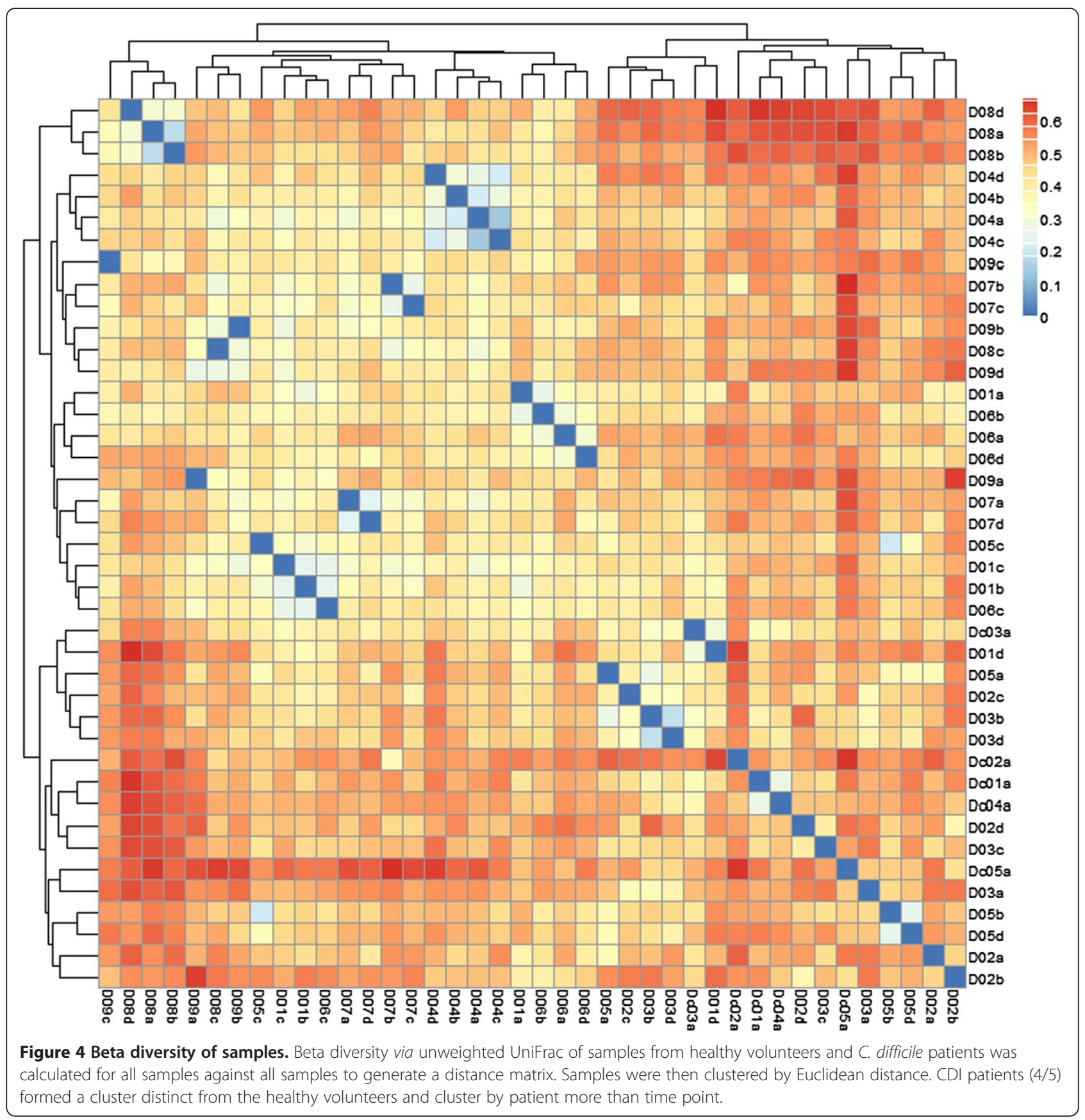

tract, we did not evaluate the upper gut microbiota, an area of particular interest given the proximity to the acid-producing stomach. Further study in this area is needed.

Kanno et al. administered omeprazole to mice and found dose-dependent changes in fecal microbial communities [26]. In contrast to the study of Garcia-Mazcorro, they found a higher abundance of all groups of fecal bacteria with the exception of Bifidobacterium during omeprazole use. Because it is unlikely that omeprazole reaches the colon intact, it was speculated that the microbial changes observed in the feces relate to an increase in bacterial load entering the colon or to changes in dietary protein reaching the colon given the role of gastric acid in the early stage of protein digestion.

Direct effects of PPIs on gut microbes may provide an explanation for the observed changes to community structure and species diversity during treatment, and may also provide a potential mechanism whereby PPIs increase the risk of CDI. Bacterial counts of many species increase in 
the setting of gastric hypochlorhydria. In contrast, omeprazole can inhibit the growth of many gut microbial species [31]. Loss of microbial diversity is a consistent feature in CDI patients [32] and animal models of antibiotic-mediated CDI [33]. In vivo antibiotic treatment leads to an increase in gut sialic acid, a favored catabolite of $C$. difficile whose availability is strongly associated with $C$. difficile bacterial load [34]. The published literature provides strong evidence for the role of nutrient competition between the gut microbiome and $C$. difficile in utilization of available amino acids [35], monomeric glucose, $N$-acetylglucosamine, and sialic acids [36]. Although disruption of a healthy gut microbiome can create an environment conducive to $C$. difficile proliferation, the specific changes in host and microbial factors that directly lead to $C$. difficile invasion in humans remain unclear.

In addition to eliminating nutrient competitors, PPI use may directly affect gene expression across metabolic pathways in favor of $C$. difficile growth. We detected a significant decrease in enrichment of the KEGG pathway "glycine-serine-threonine metabolism" in CDI subjects compared to the healthy subjects. This pathway combines the synthesis of threonine and serine from separate pathways and the final production of glycine from either amino acid [37]. Glycine is a well-known enhancer of $C$. difficile spore germination [38]. Glycine and threonine supplementation has been shown to increase in vitro bacterial load and together lead to markedly increased $C$. difficile toxin production [39]. Given these effects, the reduction of enrichment of glycine-serine-threonine in CDI is unexpected, and future work may attempt to directly quantitate amino acid concentrations in the gut in CDI subjects and GI-healthy subjects. The lack of significant changes in enrichment of this pathway in healthy subjects is expected given the lack of adverse effects reported by the healthy subjects. Further study is warranted to investigate the premise that PPI use can alter production of metabolites that serve as $C$. difficile germinants, growth factors, and toxin production enhancers.

Specific bile salts and glycine act as co-germinants of $C$. difficile spores, converting them to vegetative (infectious) forms. In vivo and independent of C. difficile, glycine appears to alter the ratio of deconjugated (primary bile salts) to conjugated (secondary bile salts) in the intestine [22]. In contrast, secondary bile salts such as deoxycholate prevent vegetative growth $[38,40]$. After severe disruption of gut microbial populations by PPI-induced hypochlorhydria, secondary bile salts are significantly reduced, allowing unrestricted vegetative growth [41]. Chenodeoxycholate, a primary bile salt, inhibits germination and protects the small intestine by inhibiting production of vegetative forms, until spores reach the anaerobic environment of the large bowel [42]. These conditions are reversed by cholestyramine, which binds bile salts that stimulate spore germination [43].

The direct effect of PPIs on $C$. difficile can be divided into effects on spore germination, effects on growth, and effects on toxin production. A recent report found that $C$. difficile spore germination in gastric contents was increased by nutrient supplementation as opposed to PPI use but did not compare germination efficacy with and without PPI [44]. No research is currently available to describe the effect of PPI on $C$. difficile spore germination or vegetative growth, but PPIs have a notable effect on toxin production of vegetative $C$. difficile. In vitro, some strains of $C$. difficile possess enhanced toxin production in the presence of omeprazole, independent of final pH [45]. Strain-level variations in enhanced toxin production of omeprazole are likely to be an explanation of why PPI use is not as strongly associated with CDI as other well-known risk factors.

\section{Conclusions}

In conclusion, we have shown that PPI use in humans reduces microbial diversity, a condition found in subjects with CDI. A follow-up study with a greater number of recruited subjects will improve our confidence in this finding, a necessary precursor to future studies. Further investigation into the role of PPIs in altering metabolism in gut bacteria is likely to solidify our understanding of PPI-mediated changes within the gut microbiome and provide additional insight into the association between prolonged PPI usage and CDI acquisition. Finally, additional research should be performed in an effort to mitigate the loss of microbial diversity consequent to PPI use.

\section{Methods}

\section{Ethics statement}

The study was approved by the Mayo Clinic Institutional Review Board (\#13-000180) and registered on ClinicalTrials. gov (NCT01822977). Written informed consent was provided by all individuals enrolled.

\section{Study participants}

Study participants were recruited at Mayo Clinic in Arizona. Ten volunteers without acute or chronic GI symptoms or conditions and five adult patients experiencing their first episode of CDI were enrolled. Healthy subjects were screened for current or chronic GI symptoms using a 16-item questionnaire. Only those with an absence of symptoms were eligible to participate. Exclusion criteria for healthy volunteers included prior surgery altering the esophagus, stomach, and intestine; chronic daily use of medications affecting GI secretion or motor function; the presence of any GI-motility affecting systemic diseases or untreated psychiatric disease; and pregnancy. Use of the following medications was prohibited: antibiotics within 2 months of the start of the study, probiotics within 2 weeks, and chronic use of medications 
that alter gastric acid (PPI, histamine-2-receptor antagonists) or motility (prokinetic agents, narcotic analgesics, laxatives, anticholinergics, antidiarrheals). Those on a stable dose of daily fiber were allowed to participate. In the $C$. difficile group, only patients experiencing their first episode of CDI who had not yet been treated were eligible to participate.

\section{Study design}

Following consent, a fresh stool sample was collected from the ten healthy subjects (T0). These ten subjects were then randomly assigned to receive omeprazole $20 \mathrm{mg}$ once daily $(n=5)$ or twice daily $(n=5)$ for 28 days. Stool samples were collected after 7 (T7) and 28 (T28) days of omeprazole use. A final stool sample was collected 1 month after discontinuation of omeprazole (T56). Additionally, fresh stool samples were collected from five treatment-naïve CDI subjects before commencing treatment.

\section{Sample collection and storage}

Subject stool samples were passed into a stool collection device at the subject's home in the morning, with the subject transferring a portion into a specimen container. The specimen container was brought to the Mayo Clinic in Arizona study coordinator fresh within 6 hours of passing. C. difficile samples were taken from consented patients clinical specimens after $C$. difficile testing was completed. At the Mayo Clinic in Arizona, the specimen was frozen at $-80^{\circ} \mathrm{C}$ before being shipped to the Mayo Clinic in Rochester in an insulated container with dry ice. At the Mayo Clinic in Rochester, samples were stored at $-80^{\circ} \mathrm{C}$ until DNA extraction.

\section{DNA extraction and library preparation}

DNA was extracted from samples using the MoBio PowerSoil Kit (MoBio Laboratories, Carlsbad, CA, USA). Genomic DNA (gDNA; $50 \mathrm{ng}$ ) was used as template for a polymerase chain reaction (PCR) with $0.3 \mathrm{uM} \mathrm{V3-V5} \mathrm{bar-}$ coded primers [46] targeting $357 \mathrm{~F}$ and 926R (5'AATGA TACGGCGACCACCGAGATCTACACTATGGTAATT GTCCTACGGGAGGCAGCAG3' and 5'CAAGCAGA AGACGGCATACGAGATGCCGCATTCGATXXXXXXX XXXXXCCGTCAATTCMTTTRAGT3', respectively) of the bacterial $16 \mathrm{~S}$ gene, along with Kapa HiFi Hotstart Ready Mix (Kapa Biosystem). PCR conditions were set to $95^{\circ} \mathrm{C} / 3 \mathrm{~min}, 35$ cycles of $98^{\circ} \mathrm{C} / 30 \mathrm{~s}, 70^{\circ} \mathrm{C} / 15 \mathrm{~s}$, $72^{\circ} \mathrm{C} / 15 \mathrm{~s}$, and finally $72^{\circ} \mathrm{C} / 5 \mathrm{~min}$ in a Bio-Rad $\mathrm{T} 100$ Thermal Cycler. PCR product sizes were verified using Agilent Tapestation with reaction cleanup and DNA was purified using an epMotion automated system (Eppendorf) with Agencourt AMPure PCR Purification System. Final quantitation was performed using QuBit HS dsDNA kit using the QuBit 2.0 flurometer (Life Technologies). Samples were pooled to equal concentrations and sequenced on one lane of MiSeq at the Mayo Genomics Facility using MiSeq
Reagent Kit v2 $(2 \times 250$ reads, 500-cycles) (Illumina Inc., San Diego, CA).

\section{NGS data analysis}

Quality control of $16 \mathrm{~S}$ reads can strongly effect ecological diversity metrics such as alpha- and beta-diversity, with filtering of low quality reads playing an important role in reducing the count of spurious OTU detection [47]. Briefly, after quality-filtering using Trimmomatic, paired $16 \mathrm{~S}$ reads were de-replicated and singletons and chimeras were removed and clustered by similarity with representative sequence selection with USEARCH, part of the UPARSE package [48]. Benchmarks by the author of the UPARSE package against mock OTU sets suggest that UPARSE better approximates actual species diversity due to its conservative nature. Representative sequences representing the OTUs were classified using Greengenes [49] 13_5 database with mothur 1.32.1 [50]. Clean sequences were re-mapped to the representative OTUs using USEARCH. Output files were converted to BIOM format and processed using QIIME 1.7.0 [51]. An observed species rarefaction curve was generated to measure saturation of sequencing (Additional file 2: Figure S1).

As the default Greengenes database did not possess $C$. difficile representatives, 72 sequences and appropriate taxonomy were downloaded from SILVA SSU r117 [52] by searching the online version of $\mathrm{r} 117$ for "clostridium difficile" for non-repetitive reference sequences $(\operatorname{Ref}(\mathrm{NR}))$. The sequences and taxonomic data were concatenated to the reference fasta and taxonomy files, respectively. This supplemented Greengenes 99 file was then used to re-classify the OTU representative reads from USEARCH in mothur using the classify.seqs function.

The $\mathrm{R}$ function sample was used to perform random sampling with replacement. The input used was a vector of incrementing values from 1 to a number equal to the total number of OTUs in the community matrix. For each sample, the probability of selecting any OTU was weighted by the relative abundance of that OTU in a particular sample. OTUs were then randomly selected 75,000 times for each sample, a value less than the read-count of the sample with the lowest read-count $(75,506)$. The list of randomly sampled OTUs per subject was converted into a down-sampled community matrix by taking the randomly sampled OTU counts and incrementing the rows of a blank community matrix corresponding to the randomly selected OTUs.

\section{Comparison of healthy and CDI datasets}

SourceTracker estimates the proportion of a sampled microbial community that is derived from defined source communities. Previous uses include estimating the proportion of the microbiota detected at a variety of "sinks" from various environmental "sources" [53]. SourceTracker 0.95 from Quantitative Insights Into Microbial Ecology (QIIME) 
was used on unrarefied data to determine the relatedness of various sink samples (T7, T28, T56) to sources (T0, $\mathrm{CD})$. The mapping file previously used with QIIME was appended to include the additional columns required to designate sources and sinks. The healthy subjects and CDI subjects at baseline were used as sources, with healthy subjects at 7-days, 28-days, and 1-month post-treatment treated as sinks. SourceTracker performed rarefaction of 75,000 reads with ten restarts and 100 burn-in iterations. The percent relationship between the aggregate sinks and the aggregate CDI and T0 sources were plotted on a ternary plot constructed using the $\mathrm{R}$ package vcd.

NMDS was selected to analyze the between-sample distances of the community matrix [54]. NMDS uses rank-order of pairwise distances in a distance matrix and is a more robust technique for measuring relatedness of samples [55]. NMDS analysis was used to determine variation between CDI- and PPI-consuming subjects over time using a community matrix rarefied to 75,000 reads/sample. The $\mathrm{R}$ package vegan was used for NMDS calculations; specifically, function metaMDS was used to perform the NMDS calculation, followed by shape generation with ordihull and labeling of samples via orditorp. Shapes were generated based on sample group and time point, comprising CDI subjects, healthy subjects at baseline (T0), subjects after 1 week of PPI treatment (T7), 1 month of treatment (T28), and 1 month after ceasing PPI treatment (T56).

\section{Diversity measures}

Taking biom and tree files generated from UPARSE and mothur, data were converted to tabular form and analyzed using R-3.1.0 on R-Studio 0.98. Observed species counts were calculated using function "specnumber" from package vegan. Shannon diversity was calculated using function "diversity" with index = "shannon" from package vegan. The rarefaction depth utilized for subsequent statistical analyses was 75,000 reads/sample. Chao diversity was calculated using function specpool with index = "chao" from package vegan. After rarefaction in QIIME, unweighted UniFrac $\beta$-diversity was calculated via beta_diversity.py in QIIME. The distance matrix was exported to $\mathrm{R}$ and visualized using heatmap, using Euclidean distance to cluster rows and columns.

\section{Statistical testing}

To test for normality of the data, the skewness function from R library e1071 [56] was used to evaluate the distribution of observed OTU data. Non-parametric Wilcoxon tests were chosen to evaluate statistical significance of observed differences between datasets. Between healthy subjects longitudinally paired data, one-tailed Wilcoxon signed rank was used to evaluate if observed OTU diversity decreased during PPI use and was still lower than at baseline. The Wilcoxon rank sum test with continuity correction was used to compare non-matched CDI subjects and healthy subjects at various time points. Wilcoxon rank sum was also used to compare high and low dosage groups and observed OTU counts between men and women.

\section{Synthetic metagenome prediction}

PICRUSt was used to infer metagenomes from the $16 \mathrm{~S}$ data [28]. From the $16 \mathrm{~S}$ data we extrapolated gene content based on the evolutionary distance of a detected OTU to the closest relative with a completed reference genome. Very close evolutionary relationships to an organism with a closed reference genome would improve the confidence of predicted genome content, in contrast to OTUs with a closed reference genome only available from a distant evolutionary relative. The distances of all taxa to the nearest organism with closed reference genome were summed to derive the NSTI for each sample. OTU picking was reperformed in QIIME using pick_closed_reference_otus.py. The resulting biom file was then normalized using normalize_otus.py to convert the OTU content into KEGG gene content. Pathway annotation was generated using predict_metagenomes.py with the normalized biom file as input with default output using KEGG pathway information [37]. KEGG pathway data was then exported in tabular form to R-3.1.0 in R-Studio 0.98 for subsequent analysis.

\section{Longitudinal changes}

Evaluation of longitudinal trends was performed by transforming numeric counts per OTU into presence/ absence $(1 / 0)$ for each subject's four time points (baseline, short-term PPI, long-term PPI, post-PPI recovery). Absence ("0") was strictly defined as no reads detected, with presence ("1") defined as detectable reads per OTU for a given subject. For two outcomes and four time points, this resulted in $2^{4}-1$ combinations of an OTU after subtracting the combination corresponding to the absence in all time points (0000). From a rarefied community matrix of 75,000 reads per sample, the behavior of each OTU in each subject was classified into 1 of 15 bins corresponding to combinations of presence and absence at each time point $(0001,0010,0100,1000,1111$, etc.). The number of OTUs that were classified as belonging to each bin were calculated and plotted to assess the effects of PPI usage on multiple species per person.

Separately, longitudinal changes to relative abundance after commencement of treatment were calculated by taking the $\log 10$ transform of relative abundance per taxa at each post-treatment time point for each individual and dividing by the relative abundance of that taxa at baseline. For transforms that equaled infinity, the value was fixed at +10 . For transforms equaling negative infinity, the value was fixed at -10 . 


\section{Availability of supporting data}

Sequences are available at NCBI SRA (SRA: SRP045730; BioProject: PRNJA259188). The metadata associated with the sequences can be found in the additional files (Additional file 1: Metadata).

\section{Additional files}

Additional file 1: Metadata. Table showing sample metadata, along with the number of paired reads after quality control.

Additional file 2: Supplemental Figures and Supplemental Tables. Figures include per-sample observed OTU rarefaction plots (Figure S1), rarefaction sensitivity plots (Figure S2), PPI effects on gender (Figure S3), and dosage (Figure S4). SourceTracker ternary plot describes relationship of on-PPI samples to CDI and baseline (Figure S5). Differential change at family level over time per subject presented on heatmap (Figure S6) Time-longitudinal plots for Shannon (Figure S7) and Chao

(Figure S8) diversity relative to CDI. Tables include statistics for rarefaction sensitivity analysis (Table $\mathbf{S} 1$ ) and longitudinal OTU bins per subject (Table S2). Table of statistical tests for gender effects of PPI (Table S3), KEGG pathway enrichment (Table S4), changes in enrichment for Garcia-Mazcorro taxa (Table S5) and longitudinal changes to Chao and Shannon diversity (Table S6).

\section{Abbreviations}

PPI: proton pump inhibitor; OTU: operational taxonomic unit; CDI: Clostridium difficile infection; NSTI: nearest sequenced taxon index; MAD: median absolute deviation

\section{Competing interests}

The authors declare that they have no competing interests.

\section{Authors' contributions}

Conceived and designed the experiments: JD, NC, and RO. Performed the experiments: CS. Analyzed the data: CS, NC, and JD. Contributed reagents/ materials/analysis tools: PJ. Wrote the paper: CS, JD, RO, and NC. All authors read and approved the final manuscript.

\section{Acknowledgements}

Janet Yao, DNA extraction from samples, performing $16 \mathrm{~S}$ barcoding, and amplification. Amy Duffy, clinical research coordinator at Mayo Clinic in Arizona. Kristin S. Inman, Ph.D., critical reading and editing of the manuscript. Mona Branstad, critical reading and editing of the manuscript. This work was partially supported by the Center for Individualized Medicine at Mayo Clinic in Arizona and the Minnesota Biotechnology Partnership. The funders had no role in the study design, data collection and analysis, decision to publish, or preparation of the manuscript.

\section{Author details}

Biomedical Informatics and Computational Biology, University of Minnesota-Rochester, Rochester, MN, USA. ${ }^{2}$ Center for Individualized Medicine, Mayo Clinic, Rochester, MN, USA. Institute for Genomic Biology, University of Illinois Urbana-Champaign, Urbana, IL, USA. ${ }^{4}$ Division of Infectious Diseases, Mayo Clinic, Scottsdale, AZ, USA. ${ }^{5}$ Department of Physiology and Biomedical Engineering, Mayo Clinic, Rochester, MN, USA. ${ }^{6}$ Division of Surgery Research, Mayo Clinic, Rochester, MN, USA. ${ }^{7}$ Department of Health Sciences Research, Mayo Clinic, Rochester, MN, USA. ${ }^{8}$ Division of Gastroenterology, Mayo Clinic, Scottsdale, AZ, USA.

Received: 16 August 2014 Accepted: 20 October 2014 Published: 25 November 2014

\section{References}

1. Peery AF, Dellon ES, Lund J, Crockett SD, McGowan CE, Bulsiewicz WJ, Gangarosa LM, Thiny MT, Stizenberg K, Morgan DR, Ringel Y, Kim HP, Dibonaventura MD, Carroll CF, Allen JK, Cook SF, Sandler RS, Kappelman $M D$, Shaheen NJ: Burden of gastrointestinal disease in the United States: 2012 update. Gastroenterology 2012, 143:1179-1187. e1171-1173.
2. AstraZeneca Annual Report and Form 20-F Information 2013. [http://www. astrazeneca-annualreports.com/2013/_assets/pdfs/Strategic_report.pdf]

3. Lindberg P, Brändström A, Wallmark B, Mattsson H, Rikner L, Hoffmann KJ: Omeprazole: the first proton pump inhibitor. Med Res Rev 1990, 10(1):1-54.

4. Sarzynski E, Puttarajappa C, Xie Y, Grover M, Laird-Fick H: Association between proton pump inhibitor use and anemia: a retrospective cohort study. Dig Dis Sci 2011, 56(8):2349-2353.

5. Lam JR, Schneider JL, Zhao W, Corley DA: Proton pump inhibitor and histamine 2 receptor antagonist use and vitamin B12 deficiency. JAMA 2013, 310(22):2435-2442.

6. Howden C: Vitamin B12 levels during prolonged treatment with proton pump inhibitors. J Clin Gastroenterol 2000, 30(1):29-33.

7. Florentin M, Elisaf MS: Proton pump inhibitor-induced hypomagnesemia: a new challenge. World J Nephrol 2012, 1(6):151-154.

8. Yu EW, Blackwell T, Ensrud KE, Hillier TA, Lane NE, Orwoll E, Bauer DC: Acid-suppressive medications and risk of bone loss and fracture in older adults. Calcif Tissue Int 2008, 83(4):251-259.

9. de Vries F, Cooper AL, Cockle SM, van Staa TP, Cooper C: Fracture risk in patients receiving acid-suppressant medication alone and in combination with bisphosphonates. Osteoporos Int 2009, 20(12):1989-1998.

10. Lo W-K, Chan WW: Proton pump inhibitor use and the risk of small intestinal bacterial overgrowth: a meta-analysis. Clin Gastroenterol Hepatol 2013, 11:483-490.

11. Lombardo L, Foti M, Ruggia O, Chiecchio A: Increased incidence of small intestinal bacterial overgrowth during proton pump inhibitor therapy. Clin Gastroenterol Hepatol 2010, 8(6):504-508.

12. Ali T, Roberts DN, Tierney WM: Long-term safety concerns with proton pump inhibitors. Am J Med 2009, 122(10):896-903.

13. Sheen $E$, Triadafilopoulos $G$ : Adverse effects of long-term proton pump inhibitor therapy. Dig Dis Sci 2011, 56(4):931-950.

14. Chitnis AS, Holzbauer SM, Belflower RM, Winston LG, Bamberg WM, Lyons C, Farley MM, Dumyati GK, Wilson LE, Beldavs ZG, Dunn JR, Gould LG, MacCannell DR, Gerding DN, McDonald LC, Lessa FC: Epidemiology of community-associated Clostridium difficile infection, 2009 through 2011. JAMA Intern Med 2013, 173:1359-1367.

15. Barletta JF, El-Ibiary SY, Davis LE, Nguyen B, Raney CR: Proton pump inhibitors and the risk for hospital-acquired Clostridium difficile infection. Mayo Clin Proc 2013, 88:1085-1090.

16. Freedberg DE, Salmasian H, Friedman C, Abrams JA: Proton pump inhibitors and risk for recurrent Clostridium difficile infection among inpatients. Am J Gastroenterol 2013, 108:1794-1801.

17. Janarthanan S, Ditah I, Adler DG, Ehrinpreis MN: Clostridium difficileassociated diarrhea and proton pump inhibitor therapy: a meta-analysis. Am J Gastroenterol 2012, 107:1001-1010.

18. Kim JW, Lee KL, Jeong JB, Kim BG, Shin S, Kim JS, Jung HC, Song IS: Proton pump inhibitors as a risk factor for recurrence of Clostridium-difficileassociated diarrhea. World J Gastroenterol 2010, 16:3573-3577.

19. Kwok CS, Arthur AK, Anibueze Cl, Singh S, Cavallazzi R, Loke YK: Risk of Clostridium difficile infection with acid suppressing drugs and antibiotics: a meta-analysis. Am J Gastroenterol 2012, 107:1011-1019.

20. FDA: FDA Drug Safety Communication: Clostridium difficile-associated diarrhea can be associated with stomach acid drugs known as proton pump inhibitors (PPIs). United States: 2012.

21. Rao A, Jump RL, Pultz NJ, Pultz MJ, Donskey CJ: In vitro killing of nosocomial pathogens by acid and acidified nitrite. Antimicrob Agents Chemother 2006, 50:3901-3904.

22. Britton RA, Young VB: Role of the intestinal microbiota in resistance to colonization by Clostridium difficile. Gastroenterology 2014, 146:1547-1553.

23. Antharam VC, Li EC, Ishmael A, Sharma A, Mai V, Rand KH, Wang GP: Intestinal dysbiosis and depletion of butyrogenic bacteria in Clostridium difficile infection and nosocomial diarrhea. J Clin Microbiol 2013, 51(9):2884-2892.

24. Chang JY, Antonopoulos DA, Kalra A, Tonelli A, Khalife WT, Schmidt TM, Young VB: Decreased diversity of the fecal microbiome in recurrent Clostridium difficile-associated diarrhea. J Infect Dis 2008, 197:435-438.

25. Schubert AM, Rogers MA, Ring C, Mogle J, Petrosino JP, Young VB, Aronoff DM, Schloss PD: Microbiome data distinguish patients with Clostridium difficile infection and non-C. difficile-associated diarrhea from healthy controls. MBio 2014, 5(3):e01021-14.

26. Kanno T, Matsuki T, Oka M, Utsunomiya H, Inada K, Magari H, Inoue I, Maekita T, Ueda K, Enomoto S, Iguchi M, Yanaoka K, Tamai H, Akimoto S, Nomoto K, Tanaka R, Ichinose M: Gastric acid reduction leads to an 
alteration in lower intestinal microflora. Biochem Biophys Res Commun 2009, 381:666-670.

27. Wallace JL, Syer S, Denou E, De Palma G, Vong L, McKnight W, Jury J, Bolla $M$, Bercik P, Collins SM, Verdu E, Ongini E: Proton pump inhibitors exacerbate NSAID-induced small intestinal injury by inducing dysbiosis. Gastroenterology 2011, 141:1314-1322

28. Langille MG, Zaneveld J, Caporaso G, McDonald D, Knights D, Reyes JA, Clemente JC, Burkepile DE, Thurber RLV, Knight R, Beiko RG, Huttenhower C: Predictive functional profiling of microbial communities using 16S rRNA marker gene sequences. Nat Biotechnol 2013, 31:814-821.

29. Martínez I, Muller CE, Walter J: Long-term temporal analysis of the human fecal microbiota revealed a stable core of dominant bacterial species. Plos One 2013, 8(7):e69621.

30. Garcia-Mazcorro JF, Suchodolski JS, Jones KR, Clark-Price SC, Dowd SE, Minamoto Y, Markel M, Steiner JM, Dossin O: Effect of the proton pump inhibitor omeprazole on the gastrointestinal bacterial microbiota of healthy dogs. FEMS Microbiol Ecol 2012, 80:624-636.

31. Jonkers D, Stobberingh E, Stockbrugger R: Omeprazole inhibits growth of Gram-positive and Gram-negative bacteria including Helicobacter pylori in vitro. J Antimicrob Chemother 1996, 37:145-150.

32. Pérez-Cobas AE, Artacho A, Ott SJ, Moya A, Gosalbes MJ, Latorre A: Structural and functional changes in the gut microbiota associated to Clostridium difficile infection. Front Microbiol 2014, 5:335.

33. Chen X, Katchar K, Goldsmith JD, Nanthakumar N, Cheknis A, Gerding DN, Kelly CP: A mouse model of Clostridium difficile-associated disease. Gastroenterology 2008, 135(6):1984-1992.

34. Ng KM, Ferreyra JA, Higginbottom SK, Lynch JB, Kashyap PC, Gopinath S, Naidu N, Choudhury B, Weimer BC, Monack DM, Sonnenburg JL: Microbiota-liberated host sugars facilitate post-antibiotic expansion of enteric pathogens. Nature 2013, 502(7469):96-99.

35. Yamamoto-Osaki T, Kamiya S, Sawamura S, Kai M, Ozawa A: Growth inhibition of Clostridium difficile by intestinal flora of infant faeces in continuous flow culture. J Med Microbiol 1994, 40(3):179-187.

36. Wilson $\mathrm{KH}$, Perini F: Role of competition for nutrients in suppression of Clostridium difficile by the colonic microflora. Infect Immun 1988, 56:2610-2614

37. Kanehisa M: Kyoto encyclopedia of genes and genomes. Nucleic Acids Res 2000, 28:27-30.

38. Sorg J, Sonenshein AL: Bile salts and glycine as cogerminants for Clostridium difficile spores. J Bacteriol 2008, 190(7):2505-2512.

39. Yamakawa K, Kamiya S, Meng XQ, Karasawa T, Nakamura S: Toxin production by Clostridium difficile in a defined medium with limited amino acids. J Med Microbiol 1994, 41:319-323.

40. Howerton A, Ramirez N, Abel-Santos E: Mapping interactions between germinants and Clostridium difficile spores. J Bacteriol 2011, 193(1):274-282.

41. Theisen J, Nehra D, Citron D, Johansson J, Hagen JA, Crookes PF, DeMeester SR, Bremner CG, DeMeester TR, Peters JH: Suppression of gastric acid secretion in patients with gastroesophageal reflux disease results in gastric bacterial overgrowth and deconjugation of bile acids. J Gastrointest Surg 2000, 4:50-54.

42. Sorg JA, Sonenshein A: Inhibiting the initiation of Clostridium difficile spore germination using analogs of chenodeoxycholic acid, a bile acid. J Bacteriol 2010, 192(19):4983-4990.

43. Giel JL, Sorg J, Sonenshein AL, Zhu J: Metabolism of bile salts in mice influences spore germination in Clostridium difficile. PLoS One 2010, 5(1):e8740.

44. Nerandzic MM, Pultz MJ, Donskey CJ: Examination of potential mechanisms to explain the association between proton pump inhibitors and Clostridium difficile infection. Antimicrob Agents Chemother 2009, 53:4133-4137

45. Stewart DB, Hegarty JP: Correlation between virulence gene expression and proton pump inhibitors and ambient $\mathrm{pH}$ in Clostridium difficile: results of an in vitro study. J Med Microbiol 2013, 62:1517-1523.

46. Caporaso JG, Lauber CL, Walters WA, Berg-Lyons D, Huntley J, Fierer N, Owens SM, Betley J, Fraser L, Bauer M: Ultra-high-throughput microbial community analysis on the Illumina HiSeq and MiSeq platforms. ISME $J$ 2012, 6(8):1621-1624.

47. Bokulich NA, Subramanian S, Faith JJ, Gevers D, Gordon Jl, Knight R, Mills DA, Caporaso JG: Quality-filtering vastly improves diversity estimates from Illumina amplicon sequencing. Nat Methods 2013, 10:57-59.
48. Edgar RC: UPARSE: highly accurate OTU sequences from microbial amplicon reads. Nat Methods 2013, 10:996-998.

49. DeSantis TZ, Hugenholtz P, Larsen N, Rojas M, Brodie EL, Keller K, Huber T, Dalevi D, Hu P, Andersen GL: Greengenes, a chimera-checked 16S rRNA gene database and workbench compatible with ARB. Appl Environ Microbiol 2006, 72:5069-5072.

50. Schloss PD, Westcott SL, Ryabin T, Hall JR, Hartmann M, Hollister EB, Lesniewski RA, Oakley BB, Parks DH, Robinson CJ, Sahl JW, Stres B, Thallinger GG, Van Horn DJ, Weber CF: Introducing mothur: open-source, platform-independent, community-supported software for describing and comparing microbial communities. Appl Environ Microbiol 2009, 75:7537-7541.

51. Caporaso JG, Kuczynski J, Stombaugh J, Bittinger K, Bushman FD, Costello EK, Fierer N, Peña AG, Goodrich JK, Gordon Jl, Huttley GA, Kelley ST, Knights D, Koenig JE, Ley RE, Lozupone CA, McDonald D, Muegge BD, Pirrung M, Reeder J, Sevinsky JR, Turnbaugh PJ, Walters WA, Widmann J, Yatsunenko T, Zaneveld J, Knight R: QIIME allows analysis of high-throughput community sequencing data g. Nat Methods 2010, 7:335-336.

52. Quast C, Pruesse E, Yilmaz P, Gerken J, Schweer T, Yarza P, Peplies J, Glöckner FO: The SILVA ribosomal RNA gene database project: improved data processing and web-based tools. Nucleic Acids Res 2013, 41(D1):D590-D596.

53. Knights D, Kuczynski J, Charlson ES, Zaneveld J, Mozer MC, Collman RG, Bushman FD, Knight R, Kelley ST: Bayesian community-wide cultureindependent microbial source tracking. Nat Methods 2011, 8:761-763.

54. Faith DP, Minchin PR, Belbin L: Compositional dissimilarity as a robust measure of ecological distance. Vegetatio 1987, 69:57-68.

55. Ramette A: Multivariate analyses in microbial ecology. FEMS Microbiol Ecol 2007, 62(2):142-160.

56. Meyer D, Dimitriadou E, Hornik K, Weingessel K, Leisch L: e1071: Misc Functions of the Department of Statistics (e1071), TU Wien. R package version 1.6-3; 2014.

doi:10.1186/2049-2618-2-42

Cite this article as: Seto et al:: Prolonged use of a proton pump inhibitor reduces microbial diversity: implications for Clostridium difficile susceptibility. Microbiome 2014 2:42.

\section{Submit your next manuscript to BioMed Central and take full advantage of:}

- Convenient online submission

- Thorough peer review

- No space constraints or color figure charges

- Immediate publication on acceptance

- Inclusion in PubMed, CAS, Scopus and Google Scholar

- Research which is freely available for redistribution
C) Biomed Central 